International Journal of Pure and Applied Mathematics

Volume $91 \quad$ No. 4 2014, 505-514

ISSN: 1311-8080 (printed version); ISSN: 1314-3395 (on-line version)

url: http://www.ijpam.eu

doi: http://dx.doi.org/10.12732/ijpam.v91i4.7

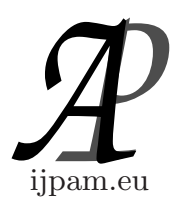

\title{
ON THE WAVE BREAKING PHENOMENON OF WEAKLY DISSIPATIVE VAKHNENKO EQUATION
}

\author{
Shengqi Yu \\ School of Xinglin, School of Sciences \\ Nantong University \\ Nantong, Jiangsu, 226007, P.R. CHINA
}

\begin{abstract}
In this paper, we study the Vakhnenko equation with linear dissipation, which can be derived from the evolution of shallow water waves on a rotating fluid in the limit of small high-frequency dissipation. We show that the wave breaking phenomenon is affected by the dissipative parameters, we also determine the exact blow-up rate of wave breaking solutions.
\end{abstract}

AMS Subject Classification: 35G25, 35L05, 35B30, 35L55

Key Words: Vakhnenko equation, wave breaking

\section{Introduction}

The Ostrovsky equation [4]

$$
\left(u_{t}+u u_{x}-\beta u_{x x x}\right)_{x}=\gamma u,
$$

with $\beta, \gamma \in \mathbb{R}$ and $u(t, x): \mathbb{R}_{+} \times \mathbb{R} \mapsto \mathbb{R}$, was derived to model small-amplitude long waves in a rotating fluid of finite depth. Its colsest relative is the wellknown Korteweg-de Vries equation (corresponds to $\gamma=0$ ). The additional term on the right hand side of the Ostrovsky equation represents the Coriolis force. Mathematical properties of the equation, such as the local and global well-posedness in energy space $[3,6]$, the convergence of solutions in the limit

Received: December 13, 2013

(c) 2014 Academic Publications, Ltd. url: www.acadpubl.eu 
of Korteweg-de Vries equation, and the stability of solitary waves were studied recently in many details.

When we consider the limit of no high-frequency dissipation $(\beta=0)$, the Ostrovsky equation can be rewritten as

$$
\left(u_{t}+u u_{x}\right)_{x}=\gamma u
$$

where $x$ is considered either on a circle or on an infinite line. We name it as the Vakhnenko equation.

In general, energy dissipation is inevitable in the real world [7], In this paper, we shall investigate the wave breaking phenomenon of the following linear dissipative Ostovsky-Hunter equation:

$$
\left\{\begin{array}{l}
\left(u_{t}+u u_{x}+\lambda u\right)_{x}=\gamma u, \quad t>0 \\
u(0, x)=u_{0}(x)
\end{array}\right.
$$

where $x$ is considered either on a circle or on an infinite line. $\lambda u_{x}$ is the linear dissipation term, and $\lambda>0$ is a dispersive constant. Using the integral estimates and the method of characteristics similar to analysis of the CamassaHolm equation and the Degasperis-Procesi equation, we find various sufficient conditions for the wave breaking and analyze the impact of the dissipative parameter on the wave breaking phenomenon, we claim that the smaller the dissipative parameter is, the easier the solution blows up. We also specify the blow-up rate at which the wave break by using the method of characteristics.

The rest of the paper is organized as follows. Section 2 gives a sufficient condition for the wave breaking in a periodic domain. The blow-up rate of the wave breaking is studied in Section 2 based on the method of characteristics. Similar wave breaking results on an infinite line are reported in Section 3.

\section{Wave Breaking and Blow-Up Phenomenon in a Periodic Domain}

Let $t>0$ and $\mathbb{S}$ denote a circle of unit length. Local well-posedness of problem (1.1) with initial data $u_{0} \in H^{s}(\mathbb{S}), s \geq 2$ can be obtained by using the work of Schäfer and Wayne [5] who studied a very similar short-pulse equation on an infinite line. More precisely, we can obtain the following local well-posedness result.

Lemma 1. Assume that $u_{0} \in H^{s}(\mathbb{S}), s \geq 2$ and $\int_{\mathbb{S}} u_{0}(x) \mathrm{d} x=0$. Then there exists a maximal existence time $T=T\left(u_{0}\right)>0$ and an unique solution 
$u(t, x)$ to the Cauchy problem (1.1) such that

$$
u(t, x) \in C\left([0, T) ; H^{s}(\mathbb{S})\right) \cap C^{1}\left([0, T) ; H^{s-1}(\mathbb{S})\right)
$$

with the following two quantities

$$
\int_{\mathbb{S}} u(t, x) \mathrm{d} x=0, t \in[0, T)
$$

and

$$
\int_{\mathbb{S}} u^{2}(t, x) \mathrm{d} x=\exp \{-2 \lambda t\} \int_{\mathbb{S}} u_{0}^{2}(x) \mathrm{d} x, t \in[0, T) .
$$

Moreover, the solution depends continuously on the initial data, i.e., the mapping $u_{0} \mapsto u: H^{s}(\mathbb{S}) \rightarrow C\left([0, T) ; H^{s}(\mathbb{S})\right) \cap C^{1}\left([0, T) ; H^{s-1}(\mathbb{S})\right)$ is continuous. The maximal existence time $T$ is independent of $s \geq 2$ in the following sense: if $u_{0}(x) \in H^{s}(\mathbb{S}) \cap H^{s^{\prime}}(\mathbb{S})$ for $s, s^{\prime} \geq 2$ and $s \neq s^{\prime}$, then

$$
\begin{array}{r}
u(t, x) \in C\left([0, T) ; H^{s}(\mathbb{S})\right) \cap C^{1}\left([0, T) ; H^{s-1}(\mathbb{S})\right), \\
u(t, x) \in C\left(\left[0, T^{\prime}\right) ; H^{s}(\mathbb{S})\right) \cap C^{1}\left(\left[0, T^{\prime}\right) ; H^{s^{\prime}-1}(\mathbb{S})\right)
\end{array}
$$

with the same $T^{\prime}=T$.

With the application of Lemma 1 and energy estimate method, we can derive the following wave breaking criterion.

Lemma 2. Given $u_{0} \in H^{s}(\mathbb{S}), s \geq 3 / 2$, and $u(t, x)$ be a solution of the Cauchy problem (1.1) guaranteed by Lemma 1. Then the corresponding solution to (1.1) blows up in a finite time if and only if

$$
\limsup _{t \rightarrow T, 0 \leq \tau \leq t}\left\|u_{x}(x, \tau)\right\|_{L^{\infty}(\mathbb{R})}=+\infty,
$$

Next, we present the main theorem of this section, i.e., the following sufficient condition for the wave breaking in the Vakhnenko equation with the linear dissipation (1.1).

Theorem 1. Assume that $u_{0} \in H^{s}(\mathbb{S}), s \geq 2$ and $\int_{\mathbb{S}} u_{0}(x) \mathrm{d} x=0$. If $u_{0}$ satisfies further

$$
\int_{\mathbb{S}}\left(u_{0}^{\prime}(x)\right)^{3} \mathrm{~d} x<\left[\frac{1}{4}\left(3 \lambda-\sqrt{9 \lambda^{2}+24 \gamma Q_{0}}\right)\right]^{3},
$$

where $Q_{0}=\left\|u_{0}\right\|_{L^{2}}$, then the solution $u(t, x)$ of the Cauchy problem (1.1) in Lemma 1 blows up in finite time. 
Proof. Let $T>0$ be the maximal existence time of the solution $u(t, x)$ in Lemma 1, then we obtain by Eq. (1.1) and Lemma 1 that

$$
\begin{aligned}
\frac{\mathrm{d}}{\mathrm{d} t} \int_{\mathbb{S}} u_{x}^{3} \mathrm{~d} x & =3 \int_{\mathbb{S}} u_{x}^{2}\left(-u_{x}^{2}-u u_{x x}+\gamma u-\lambda u_{x}\right) \mathrm{d} x \\
& =-2 \int_{\mathbb{S}} u_{x}^{4} \mathrm{~d} x+3 \gamma \int_{\mathbb{S}} u u_{x}^{2} \mathrm{~d} x-3 \lambda \int_{\mathbb{S}} u_{x}^{3} \mathrm{~d} x \\
& \leq-2\left\|u_{x}\right\|_{L^{4}}^{4}+3 \gamma\left\|u_{0}\right\|_{L^{2}}\left\|u_{x}\right\|_{L^{4}}^{2}-3 \lambda \int_{\mathbb{S}} u_{x}^{3} \mathrm{~d} x \\
& \leq-2\left(\left\|u_{x}\right\|_{L^{4}}^{2}-\frac{3 \gamma}{4}\left\|u_{0}\right\|_{L^{2}}\right)^{2}+\frac{9}{8} \gamma^{2}\left\|u_{0}\right\|_{L^{2}}^{2}-3 \lambda \int_{\mathbb{S}} u_{x}^{3} \mathrm{~d} x(2.1)
\end{aligned}
$$

where we have used Holder's inequality in the above estimates. The application of Holder's inequality again yields

$$
\left\|u_{x}\right\|_{L^{3}} \leq\left\|u_{x}\right\|_{L^{4}}
$$

Denote $V(t):=\int_{\mathbb{S}} u_{x}^{3} \mathrm{~d} x$ for all $t \in[0, T), Q_{0}:=\left\|u_{0}\right\|_{L^{2}}$, then we have

$$
\left\|u_{x}\right\|_{L^{4}}^{2}-\frac{3 \gamma}{4}\left\|u_{0}\right\|_{L^{2}} \geq\left\|u_{x}\right\|_{L^{3}}^{2}-\frac{3 \gamma}{4}\left\|u_{0}\right\|_{L^{2}} \geq|V|^{\frac{2}{3}}-\frac{3 \gamma}{4} Q_{0}
$$

thus it follows from (2.1) that

$$
\frac{\mathrm{d} V}{\mathrm{~d} t} \leq-2\left(|V|^{\frac{2}{3}}-\frac{3 \gamma Q_{0}}{4}\right)^{2}+\frac{9}{8} \gamma^{2} Q_{0}^{2}-3 \lambda V(t),
$$

where the right hand side is negative at $t=0$. By the continuation argument, $V(t)$ is decreasing on $[0, T)$ so that $V(t) \leq V(0)<0$. We need to prove that $T$ is finite and $\lim _{t \rightarrow T} V(t)=-\infty$. For this purpose, we set $W(t)=|V(t)|^{\frac{1}{3}}$ and we obtain that

$$
\frac{\mathrm{d} W}{\mathrm{~d} t} \geq \frac{2}{3}\left(W^{2}-\frac{3}{2} \gamma Q_{0}\right)-\lambda W,
$$

where the right hand side is positive at $t=0$. By the comparison principle $W(t) \geq \underline{W}(t)$ for all $t \in[0, T)$, where $\underline{W}(t)$ solves the following equation

$$
\left\{\begin{array}{l}
\underline{\dot{W}}(t)=\frac{2}{3}\left(\underline{W}^{2}-\frac{3}{2} \gamma Q_{0}\right)-\lambda \underline{W} \\
\underline{W}(0)=W(0),
\end{array}\right.
$$

Since by the assumption $W(0)>\frac{1}{4}\left(\sqrt{9 \lambda^{2}+24 \gamma Q_{0}}-3 \lambda\right)$, there is a finite time $\underline{T} \in(0, \infty)$ such that $\lim _{t \rightarrow \underline{T}} \underline{W}(t)=+\infty$. 
Next, we shall give more insight into the blow-up mechanism for the wave breaking solutions to (1.1). We shall investigate the blow-up rate of the wave breaking solutions of Cauchy problem (1.1), which we may rewrite as

$$
\begin{cases}u_{t}+u u_{x}+\lambda u=\gamma \partial_{x}^{-1} u, & x \in \mathbb{S}, t>0 \\ u(0, x)=u_{0}(x), & x \in \mathbb{S},\end{cases}
$$

where $\partial_{x}^{-1}$ is the mean zero anti-derivative in the sense

$$
\partial_{x}^{-1} u=\int_{0}^{x} u\left(t, x^{\prime}\right) \mathrm{d} x^{\prime}-\int_{\mathbb{S}} \int_{0}^{x} u\left(t, x^{\prime}\right) \mathrm{d} x^{\prime} \mathrm{d} x .
$$

we use the method of characteristics, which is also used in similar contents by [1] . Let $T>0$ be maximal time of existence of the solution $u(t, x)$ of Cauchy problem (2.2) in Lemma 1 with the initial data $u_{0} \in H^{s}(\mathbb{S})$ for $s \geq 2$, for all $t \in[0, T)$ and $\xi \in \mathbb{S}$, define

$$
x=X(t, \xi), \quad u(t, x)=U(t, \xi), \quad \partial_{x}^{-1} u(t, x)=G(t, \xi)
$$

so that

$$
\left\{\begin{array} { l } 
{ \dot { X } ( t ) = U , } \\
{ X ( 0 ) = \xi , }
\end{array} \quad \left\{\begin{array}{l}
\dot{U}(t)=\gamma G-\lambda U \\
U(0)=u_{0}(\xi)
\end{array}\right.\right.
$$

where dots denote derivatives with respect to time $t$ on a particular characteristic $x=X(t, \xi)$ for fixed $\xi \in \mathbb{S}$. Applying the classical results in the theory of ODE, we obtain the following useful result

Lemma 3. Let $u_{0} \in H^{s}(\mathbb{S}), s \geq 2$ and $T>0$ be the maximal existence time of the solution $u(t, x)$ in Lemma 1. Then there exists a unique solution $X(t, \xi) \in C^{1}([0, T) \times \mathbb{S})$ to the initial problem (2.2). Moreover, the mapping $X(t, \cdot): \mathbb{S} \rightarrow \mathbb{R}$ is an increasing diffeomorphism with

$$
\partial_{\xi} X(t, \xi)=\exp \left(\int_{0}^{t} u_{x}(s, X(s, \xi)) \mathrm{d} s\right)>0, \forall t \in[0, T), \forall x \in \mathbb{S} .
$$

Lemma 4. Let $u_{0} \in H^{s}(\mathbb{S}), s \geq 2$ and $T>0$ be the maximal existence time of the solution $u(t, x)$ in Lemma 1. Then the solution to problem (1.1) satisifies

$$
\sup _{s \in[0, t)}\|u(s, \cdot)\|_{L^{\infty}} \leq \exp \{-\lambda t\}\left(\frac{\gamma}{\lambda}\left\|u_{0}\right\|_{L^{2}}+\left\|u_{0}\right\|_{L^{\infty}}\right),
$$


Theorem 2. Let $u_{0} \in H^{s}(\mathbb{S}), s \geq 2$ and assume that there exists a $x_{0} \in \mathbb{S}$ such that

$$
u_{0}^{\prime}\left(x_{0}\right) \leq-\frac{1}{2} \lambda-\frac{1}{2} \sqrt{\lambda^{2}+4 \gamma\left(\left\|u_{0}\right\|_{L^{\infty}}+\frac{\gamma}{\lambda}\left\|u_{0}\right\|_{L^{2}}\right)},
$$

then the corresponding solution of (1.1) blows up in finite time.

Proof. Define $V(t, \xi)=u_{x}(t, X(t, \xi))$, by Lemmas 1 and $3, V(t, \xi)$ is absolutely continuous on $[0, T) \times \mathbb{S}$ and almost everywhere differentiable on $[0, T) \times \mathbb{S}$, so that

$$
\dot{V}=\left.\left(u_{t x}+u u_{x x}\right)\right|_{x=X(t, \xi)}=\left.\left(\gamma u-u_{x}^{2}-\lambda u_{x}\right)\right|_{x=X(t, \xi)}=-V^{2}+\gamma u-\lambda V
$$

a.e. $\xi \in \mathbb{S}, t \in[0, T)$. We obtain by Lemma 4 the following a prior estimate

$$
\dot{V} \leq-V^{2}-\lambda V+\gamma\left(\left\|u_{0}\right\|_{L^{\infty}}+\frac{\gamma}{\lambda}\left\|u_{0}\right\|_{L^{2}}\right) \text {, a.e. } \xi \in \mathbb{S}, t \in[0, T) .
$$

Set

$$
\begin{gathered}
\alpha^{2}=\gamma\left(\left\|u_{0}\right\|_{L^{\infty}}+\frac{\gamma}{\lambda}\left\|u_{0}\right\|_{L^{2}}\right) \\
\frac{\mathrm{d} V}{\mathrm{dt}} \leq-V^{2}-\lambda V+\alpha^{2} \\
=-\frac{1}{4}\left(2 V(t)+\lambda-\sqrt{\lambda^{2}+4 \alpha^{2}}\right)\left(2 V(t)+\lambda+\sqrt{\lambda^{2}+4 \alpha^{2}}\right) .
\end{gathered}
$$

From the hypothesis, we have $V(0)<-\frac{1}{2} \lambda-\frac{1}{2} \sqrt{\lambda^{2}+4 \alpha^{2}}$, and thus $\left.\frac{\mathrm{d} V}{d t}\right|_{t=0}<0$. By the continuity with respect to $t$ of $V(t)$, we have $\frac{\mathrm{d} V}{d t}<0$ for all $t \in[0, T)$. Therefore $V(t)<-\frac{1}{2} \lambda-\frac{1}{2} \sqrt{\lambda^{2}+4 \alpha^{2}}$ for all $t \in[0, T)$. Thus we can solve the above inequality to obtain

$$
\frac{2 V(0)+\lambda+\sqrt{\lambda^{2}+4 \alpha^{2}}}{2 V(0)+\lambda-\sqrt{\lambda^{2}+4 \alpha^{2}}} \exp \left\{\sqrt{\lambda^{2}+4 \alpha^{2}} t\right\}-1 \leq \frac{2 \sqrt{\lambda^{2}+4 \alpha^{2}}}{2 V(t)+\lambda-\sqrt{\lambda^{2}+4 \alpha^{2}}} \leq 0 .
$$

Since

$$
0<\frac{2 V(0)+\lambda+\sqrt{\lambda^{2}+4 \alpha^{2}}}{2 V(0)+\lambda-\sqrt{\lambda^{2}+4 \alpha^{2}}}<1,
$$

there exists $T$ satisfying

$$
T \leq \frac{1}{\sqrt{\lambda^{2}+4 \alpha^{2}}} \ln \left(\frac{2 V(0)+\lambda+\sqrt{\lambda^{2}+4 \alpha^{2}}}{2 V(0)+\lambda-\sqrt{\lambda^{2}+4 \alpha^{2}}}\right),
$$


such that $\lim _{t \rightarrow T} V(t)=-\infty$. Hence, the theorem is proved according to Lemma 2 .

Our final result in this section mainly concerns with the blow-up rate of the wave breaking solutions to Eq. (1.1), since the method of proof is standard, we omit the detail process.

Theorem 3. Let $u_{0} \in H^{s}(\mathbb{S}), s \geq 3$, and $T>0$ be the maximal existence time of the solution $u(t, x)$ in Lemma 1. If $T$ is finite, we have

$$
\lim _{t \rightarrow T}\left((T-t) \inf _{x \in \mathbb{S}} u_{x}(t, x)\right)=-1
$$

and

$$
\lim _{t \rightarrow T}\left((T-t) \sup _{x \in \mathbb{S}} u_{x}(t, x)\right)=0 .
$$

\section{Wave Breaking and Blow-Up Phenomenon on an Infinite Line}

To extend our results on wave breaking in the Vakhnenko equation with dissipation (1.1) from a circle $\mathbb{S}$ to an infinite line $\mathbb{R}$. Consider the Cauchy problem in the form

$$
\left\{\begin{array}{l}
u_{t}+u u_{x}+\lambda u=\gamma \partial_{x}^{-1} u, \quad x \in \mathbb{R}, t>0 \\
u(0, x)=u_{0}(x), \quad x \in \mathbb{R},
\end{array}\right.
$$

where $\gamma>0$ and $\partial_{x}^{-1} u=\int_{-\infty}^{x} u\left(t, x^{\prime}\right) \mathrm{d} x^{\prime}$. To control $\partial_{x}^{-1} u$, we define $\|u\|_{\dot{H}^{-1}}:=$ $\left\|\partial_{x}^{-1} u\right\|_{L^{2}}$. The local well-posedness result for the whole line case is given by the following lemma.

Lemma 5. Assume that $u_{0} \in H^{s}(\mathbb{R}) \cap \dot{H}^{-1}(\mathbb{R}), s \geq 2$. Then there exists a maximal time $T=T\left(u_{0}\right)>0$ and an unique solution $u(t, x)$ to the Cauchy problem (3.1) such that

$$
u(t, x) \in C\left([0, T) ; H^{s}(\mathbb{R}) \cap \dot{H}^{-1}(\mathbb{R})\right) \cap C^{1}\left([0, T) ; H^{s-1}(\mathbb{R})\right)
$$

with the following quantities

$$
\int_{\mathbb{R}} u(t, x) \mathrm{d} x=0, t \in[0, T)
$$

and

$$
\int_{\mathbb{R}} u^{2}(t, x) \mathrm{d} x=e^{-2 \lambda t} \int_{\mathbb{R}} u_{0}^{2}(x) \mathrm{d} x, t \in[0, T) .
$$


Moreover, the solution depends continuously on the initial data, i.e., the mapping $\left.u_{0} \mapsto u: H^{s}(\mathbb{R}) \rightarrow C\left([0, T) ; H^{s}(\mathbb{R}) \cap \dot{H}^{-1}(\mathbb{R})\right) \cap C^{1}\left([0, T) ; H^{s-1}(\mathbb{R})\right)\right)$ is continuous.

Remark 1. The conserved quantity

$$
E=\int_{\mathbb{R}}\left[\gamma\left(\partial_{x}^{-1} u\right)^{2}+\frac{1}{3} u^{3}\right] \mathrm{d} x=\int_{\mathbb{R}}\left[\gamma\left(\partial_{x}^{-1} u_{0}\right)^{2}+\frac{1}{3} u_{0}^{3}\right] \mathrm{d} x, \quad t \in[0, T)
$$

which holds for the nondissipative case $(\lambda=0)$ does not holds here even in the exponential decaying form like (3.3), which causes some difficulty in the estimate of the norm $\|u\|_{L^{\infty}}$.

Since the Sobolev embedding $H^{3}(\mathbb{R}) \hookrightarrow C^{2}(\mathbb{R})$ is available, we can extend the blow-up criterion Lemma 2 to the whole line case, however, because the application of the Holder inequality $L^{3} \hookrightarrow L^{4}$ is not valid on $\mathbb{R}$, Theorem 1 can not be extended on an infinite line. However, we can still use the method of characteristics and extend Theorem 2 and Theorem 3 from $\mathbb{S}$ to $\mathbb{R}$.

For all $t \in[0, T)$ and $\xi \in \mathbb{R}$, we define

$$
X=X(t, \xi), u(t, x)=U(t, \xi), \partial_{x}^{-1} u(t, x)=G(t, \xi),
$$

Consider the same ODE system, Lemma 3 holds on $\mathbb{R}$, while Lemma 4 is replaced with the following lemma.

Lemma 6. Let $u_{0} \in H^{s}(\mathbb{R}) \cap \dot{H}^{-1}(\mathbb{R}), s \geq 2$. $T>0$ be the maximal existence time of the solution $u(t, x)$ in Lemma 5. Then the solution $u(t, x)$ satisfies

$$
\sup _{s \in[0, t]}\|u(s, \cdot)\|_{L^{\infty}} \leq\left\|u_{0}\right\|_{L^{\infty}}+C t+\frac{1+\lambda}{6} \gamma Q t^{2}:=P_{\lambda}(t), t \in[0, T),
$$

where $C=\left[\frac{\gamma}{2}\left(A_{0}+\gamma Q+\frac{1+\lambda}{3} Q\left\|u_{0}\right\|_{L^{\infty}}\right)\right]^{\frac{1}{2}}, A_{0}=\gamma \int_{\mathbb{R}}\left(\partial_{x}^{-1} u_{0}\right)^{2} \mathrm{~d} x+\frac{1}{3} \int_{\mathbb{R}} u_{0}^{3} \mathrm{~d} x$.

Theorem 4. Let $\varepsilon>0$ and $u_{0} \in H^{s}(\mathbb{R}) \cap \dot{H}^{-1}(\mathbb{R}), s \geq 2$. $T_{1}$ be the minimal root of the following equation:

$$
\left(\lambda^{2}+4 \gamma P_{\lambda}\left(T_{1}\right)\right)^{\frac{1}{2}}=\ln \left(1+\frac{2}{\varepsilon}\right),
$$

assume in addition there exists $x_{0} \in \mathbb{S}$, such that

$$
u_{0}^{\prime}\left(x_{0}\right) \leq-\frac{\lambda}{2}-\frac{1+\varepsilon}{2} \sqrt{\lambda^{2}+4 \gamma P_{\lambda}\left(T_{1}\right)}
$$

where the function $P_{\lambda}(t)$ is given in (3.4), then the solution given by Lemma 5 blows up in finite time $T \in\left(0, T_{1}\right)$. 
Proof. Define $W(t, \xi)=u_{x}(t, X(t, \xi))$, by Lemma 1 and Lemma $3, W(t, \xi)$ is absolutely continuous on $[0, T) \times \mathbb{R}$ and almost everywhere differentiable on $(0, T) \times \mathbb{R}$, so that

$$
\dot{W}=\left.\left(u_{t x}+u u_{x x}\right)\right|_{x=X(t, \xi)}=\left.\left(\gamma u-u_{x}^{2}-\lambda u_{x}\right)\right|_{x=X(t, \xi)}=-W^{2}+\lambda W+\gamma U,
$$

a.e. $\xi \in \mathbb{R}, t \in(0, T)$.

By Lemma 6, we obtain the apriori differential estimate

$$
\dot{W} \leq-W^{2}-\lambda W+\gamma P_{\lambda}(t), \quad \text { a.e. } \quad \xi \in \mathbb{R}, t \in(0, T) .
$$

It follows from the assumption of theorem (3.6) that, for fixed $\varepsilon>0$, there exists $\tilde{x}_{0}$, such that

$$
W\left(0, \tilde{x}_{0}\right)=-\frac{\lambda}{2}-\frac{1+\varepsilon}{2} \sqrt{\lambda^{2}+4 \gamma T_{1}}
$$

Thanks to the apriori estimate $(3.7), W(t):=W\left(t, \tilde{x}_{0}\right)$ satisfies

$$
\left\{\begin{array}{l}
\dot{W}(t) \leq-W^{2}(t)-\lambda W(t)+\gamma P_{\lambda}\left(T_{1}\right), \quad \text { a.e. } \quad t \in\left[0, T_{1}\right] \cap(0, T), \\
W(0)=-\frac{\lambda}{2}-\frac{1+\varepsilon}{2} \sqrt{\lambda^{2}+4 \gamma P_{\lambda}\left(T_{1}\right)},
\end{array}\right.
$$

Assume that $W_{+}(t)$ be the solution of the following ODE problem

$$
\left\{\begin{array}{l}
\dot{W}_{+}(t) \leq-W_{+}^{2}(t)-\lambda W_{+}(t)+\gamma P_{\lambda}\left(T_{1}\right), \quad \text { a.e. } \quad t \in\left[0, T_{1}\right] \cap(0, T), \\
W_{+}(0)=-\frac{\lambda}{2}-\frac{1+\varepsilon}{2} \sqrt{\lambda^{2}+4 \gamma P_{\lambda}\left(T_{1}\right)}
\end{array}\right.
$$

By the comparison principle for ODEs, we have

$$
W(t) \leq W_{+}(t)<0, \quad t \in\left[0, T_{1}\right] \cap[0, T) .
$$

Set $\beta=\frac{1}{2} \sqrt{\lambda^{2}+4 \gamma P_{\lambda}\left(T_{1}\right)}$, Eq.(3.8) admits an implicit solution

$$
\frac{W_{+}(t)+\frac{\lambda}{2}+\beta}{W_{+}(t)+\frac{\lambda}{2}-\beta}=\frac{W(0)+\frac{\lambda}{2}+\beta}{W(0)+\frac{\lambda}{2}-\beta} e^{2 \beta t}, \quad t \in\left[0, T_{1}\right) .
$$

If $T_{1}$ is the smallest positive root of $(3.5)$, then

$$
\frac{W_{+}(t)+\frac{\lambda}{2}+\beta}{W_{+}(t)+\frac{\lambda}{2}-\beta}=\frac{\varepsilon}{2+\varepsilon} e^{2 \beta t} \rightarrow 1, \quad \text { as } t \rightarrow T_{1}
$$

so that $\lim _{t \rightarrow T_{1}} W_{+}(t)=-\infty$, therefore, there exists $T \in\left(0, T_{1}\right)$, such that $\lim _{t \rightarrow T} W(t)=-\infty$. 


\section{Acknowledgments}

This work is partially supported by the Natural Science Foundation of Xinglin School grant-2012K103, the Collegiate Natural Science Foundation of Jiangsu Province grant-13KJB110023, 12KJB110018 and the Start Up Foundation for Doctors in Nantong University grant-12R063.

\section{References}

[1] J. Hunter, Numerical solutions of some nonlinear dispersive wave equations, Lectures in Appl. Math., 26 (1990), 301-316.

[2] S. Levandosky, Y. Liu, Stability of solitary waves of a generalized Ostrovsky equation, SIAM J. Math. Anal., 38, No. 3 (2006), 985-1011, doi:10.1137/050638722.

[3] F. Linares, A. Milanes, Local and global well-posedness for the Ostrovsky equation, J. Diff. Eqs., 222, No. 2 (2006), 325-340, doi:10.1016/j.jde.2005.07.023.

[4] L.A. Ostrovsky, Nonlinear internal waves in a rotating ocean, Okeanologia, 18 (1978), 181-191.

[5] T. Schafer, C. E. Wayne, Propagation of ultra-short optical pulses in cubic nonlinear media, Physica D, 196, No. 1-2 (2004), 90-105, doi:10.1016/j.physd.2004.04.007.

[6] V. Varlamov, Y. Liu, Cauchy problem for the Ostrovsky equation, Discr. Cont. Dyn. Syst., 10, No. 3 (2004), 731-753, doi:10.3934/dcds.2004.10.731.

[7] S.Q. Yu and M.X. Wang, On the dissipative form of Camassa-Holm equation, J. Math. Phys., 51, No. 9 (2010), Article ID 092704, 20 pages, doi:10.1063/1.3451108. 